\title{
Influence of time-dependence on failure of echelon rock joints through a novel DEM model
}

\author{
Mingjing Jiang ${ }^{1,2,3^{*}}$, Chao Sun ${ }^{2,3}$, Alfonso Rodriguez-Dono ${ }^{4,5}$, Ning Zhang ${ }^{2,3}$, \\ Jianfu Shao
}

${ }^{1}$ State Key Laboratory for Disaster Reduction in Civil Engineering, Tongji University, Shanghai, China, 200092

${ }^{2}$ Key Laboratory of Geotechnical and Underground Engineering of Ministry of Education, Tongji University, Shanghai, China, 200092

${ }^{3}$ Department of Geotechnical Engineering, College of Civil Engineering, Tongji University, Shanghai, China, 200092

${ }^{4}$ Department of Geotechnical Engineering and Geo-Sciences, Polytechnic University of Catalonia (UPC), Barcelona, Spain

${ }^{5}$ Institute of Environmental Assessment and Water Research. Spanish Council for Scientific Research (CSIC).

* Corresponding author: Dr. Mingjing Jiang, Professor of Geotechnical Engineering, 'Professor of Exceptional Rank in Tongji University’

Tel: +0086-21-65980238; Fax: +0086-21-65985210;

E-mail: mingjing.jiang@tongji.edu.cn

Chao Sun, E-mail: 1232591@tongji.edu.cn

Alfonso Rodriguez-Dono, E-mail: alfonso.rodriguez@upc.edu

Ning Zhang, E-mail: 5921qly@tongji.edu.cn

Jianfu Shao, E-mail:jian-fu.shao@polytech-lille.fr

\section{Acknowledgement}

This work is funded by the Major Project of Chinese National Programs for Fundamental Research and Development (973 Program) (Nos. 2011CB013504 and 2014CB046901), China National Funds for Distinguished Young Scientists (No. 51025932) and State Key Lab. of Disaster Reduction in Civil Engineering (No. SLDRCE14-A-04). 
Abstract: This paper investigates the time-dependent influence on the shear failure behavior of parallel rock joints in the echelon arrangement due to chemical weathering, which can be treated as a generalized time dependency of the rock material. A time-dependent parameter $\alpha$, identifying the accumulated relative mass removal of bonding material, has been implemented into a novel DEM bond contact model. This model is based on a series of mechanical test on bonded aluminum rods with different bond geometries. The numerical direct shear test results of echelon rock joints characterized by different values of $\alpha$ show that increasing time-dependent parameter $\alpha$ can lead to a lower crack initiation and peak stresses. This is accompanied by a growing ratio of the microscopic compressive-shear-torsional bond failure (CST) number of bond failures to the total number of failures, except for the case without weathering influence. High values of $\alpha$ render the material bridge a weaker part to be cut through, generating a large number of compressive-sheartorsional bond breakages along the central shear axis.

Key words: time-dependency; rock joints; DEM; chemical weathering 


\section{Introduction}

Time-dependent alterations in the physical and mechanical properties of the bedrock due to chemical weathering has arisen great attention from geological researchers and engineers (Chigira \& Oyama, 2000). These alterations cause deterioration of the rock and instability in the rock mass, which is a potential threat to large-scale rock engineering projects, e.g. high cutslope, huge bridge-anchorage, underground power stations, carbon dioxide sequestration, underground storage of radioactive waste, etc. Time-dependent behavior normally refers to creep, a continued deformation in cases of high point loads, aggressive chemistry or tensile stresses leading to time-dependent fracture growth (Dusseault \& Fordham, 1993). This means that during creep, the physical and mechanical properties of rock material change, as in the process of chemical weathering. Being a natural process under specific physico-chemical circumstances and dominant among all kinds of degradations in humid subtropical and tropical regions, chemical weathering can thus be classified as one kind of generalized time-dependent behaviors of rocks and rock masses.

In previous studies, most researchers focused on how to evaluate the weathering extent of the rock sample (e.g. Duzgoren-Aydin et al., 2002; Ohta \& Arai, 2007; Price \& Velbel, 2003) and the relationship between weathering rate and time (e.g. Hachinohe et al., 2000; Velbel, 1993; White \& Brantley, 2003). This kind of time-dependency due to weathering is too complex to fully obtain the laws for alterations of the macroscopic properties on the basis of current experimental and field data, with two processes both intrinsic to the varied minerals composing the rock material and extrinsic to the weathering environment. Some researchers tried to investigate this issue from the microscopic level (e.g. Ciantia et al., 2013; Ciantia \& Hueckel, 2013), through employing different techniques: X-ray micro-computer-tomography (MCT), scanning electron microscope (SEM) analyses and mercury intrusion porosimetry (MIP). By observing the rock material at micro scale, numerous grains with distinctive inter-particle bonds can be recognized. Consequently, the time-dependent behavior due to chemical weathering can be simplified into the process of mass dissolution and removal, finally configuring the debonding among those cemented grains (Buscarnera, 2012; Ciantia et al., 2013). This simplified debonding law can be implemented into a distinct element method (DEM) microscopic contact model, making this model a suitable tool to analyze this time-dependency of rock behavior, which is one primary motivation of this academic work.

Furthermore, discontinuities within the rock mass dominate the behavior -especially failure- of the rock mass (Tang et al., 1998; R. H. Wong \& Chau, 1998). Rock joints in the socalled echelon arrangement, characterized by the parallel and laterally displaced separated joints (Gehle \& Kutter, 2003), have been widely observed in situ. Therefore, investigation of the breakage of echelon rock joints enables understanding of the much more complex failure of the rock mass due to the large number of possible interactions among the discontinuities. Although some promising experimental and numerical results have been obtained for specimens containing non-persistent joints under different geometrical and loading conditions (e.g. Gehle \& Kutter, 2003; Ghazvinian et al., 2012; Sarfarazi et al., 2014; R. Wong et al., 2001; Zhang et al., 2006), the influence of the time-dependent factor on the failure of rock joints is not yet clear. Also, because experimental results are very sensitive to sample-preparation 
processes and boundary loading conditions, any small change in the contact conditions between sample and loading platens may result in different failure modes for the sample. Thus, a numerical approach seems to be suitable for studying crack propagation and coalescence in rock, due to the mechanical and geometrical complexity of this kind of problems.

Therefore, this paper aims to investigate the time-dependency of failure among echelon rock joints through a novel DEM model by incorporating the weathering effect in a DEM contact law. The complex time-dependency is simplified as the bond material degradation happens homogenously within the rock sample. Four scenarios with different values of the timedependent parameter are compared with each other to analyze the influence of time-dependency on the failure of the parallel joints in the echelon arrangement. Those comparisons are presented from both macroscopic and microscopic viewpoints, e.g. shear stress and displacement relationship, the number of bond failures of two types, force chains, principal stress vector and bond breakage distributions.

\section{Bond contact model incorporating weathering effect}

The conceptual bond contact model, as illustrated in Fig. 1, is characterized by the radii of two cemented disks with diameters $D_{1}$ and $D_{2}$ and the dimensions of the bond (i.e. bond width $B$ and bond thickness $H$ ). In order to introduce the slight chemical weathering effect into the DEM microscopic contact model, it is simply assumed that the chemical dissolution reactions happen only on the bonding material cementing adjacent grains instead of a complex alteration in the particle geometries. A time-dependent parameter identifying the ratio of dissolved bond mass to the initial mass $\alpha$ is implemented into the function of the bond width in a twodimensional context:

$$
B=B_{i} \times\left(1-\frac{\alpha}{\alpha_{c r i}}\right)^{2}
$$

where $B_{i}$ is the very initial width of the bond before any weathering processes take place; $\alpha_{\text {cri }}$ is the critical ratio of dissolved bond mass to the initial one, which is assumed to be $80 \%$ in the following simulation. Since the main focus of this study is how shear behavior of rock joints changes with varying microscopic time-dependent parameter $\alpha$, how $\alpha$ changes with time is not considered in this numerical work.

The mechanical responses are similar to the contact model characterized by bond rolling resistance elucidated by Jiang et al. (2006). Contact forces are assumed to be transferred through not only the bond but also the inter-particle contact, since the adjacent particles are in touch with each other, i.e. $F_{n}=F_{n}^{b}+F_{n}^{p}, F_{s}=F_{s}^{b}+F_{s}^{p}, M=M^{b}+M^{p}$, where $F_{n}^{b}, F_{s}^{b}$ and $M^{b}$ are the normal force, the shear force and the moment transferred through the bond, respectively, while $F_{n}^{p}, F_{s}^{p}$ and $M^{p}$ are the corresponding components transferred through the inter-particle contact. The initial inter-particle contact forces, existing before the bond forms, are not involved in the force distribution as bonds generate. The inter-particle mechanical responses can be formulated in the same way as the inter-particle rolling contact model, while the bond contact forces can be expressed as: 


$$
\begin{gathered}
F_{n}^{b}=\left\{\begin{array}{c}
\min \left[K_{n}^{b}\left(u_{n}-u_{n 0}\right), R_{c b}\right]\left(u_{n}-u_{n 0} \geq 0\right) \\
\min \left[K_{n}^{b}\left(u_{n 0}-u_{n}\right), R_{t b}\right]\left(u_{n}-u_{n 0}<0\right)
\end{array}\right. \\
F_{s}^{b}=\min \left[K_{s}^{b} u_{s}, R_{s b}\right] \\
M^{b}=\min \left[K_{m}^{b} \theta, R_{r b}\right]
\end{gathered}
$$

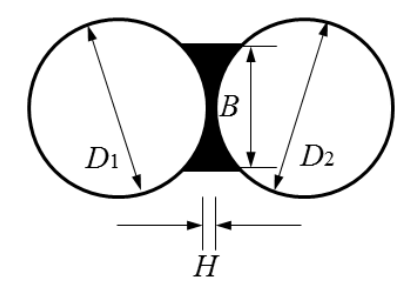

Figure 1. Schematic illustration of bonded grains

where $\min [\cdot]$ is the operator taking the minimum; $u_{n}, u_{n 0}, u_{s}, \theta$ are the overlap, the initial overlap when the bond forms, the shear displacement and the relative rotation angle, respectively; $K_{n}^{b}$, $K_{s}^{b}=K_{n}^{b} /$ Constant and $K_{m}^{b}=0.2 B^{2} K_{n}^{b}$ are the normal, shear and rolling contact stiffness, evaluated based on the experimental data of mechanical test on bonded rods; $R_{c b}, R_{t b}, R_{s b}$ and $R_{r b}$ are the normal compressive, normal tensile, shear and rolling bond strength. $K_{n}^{b}$ is related to the Young's modulus of the bonding material, $E_{b}$ :

$$
K_{n}^{b}=E_{b} \times \frac{B}{H_{a v g}}
$$

A generic bond strength envelope derived from the experimental test results of cemented aluminum rods with different bond geometries is employed in this contact model. The compressive and tensile strengths $R_{c b}, R_{t b}$ are functions of both the macroscopic strength of the bonding material and the geometries of the bonded granules:

$$
\begin{aligned}
& R_{c b}=\sigma_{c} \times B \times L \times\left(\frac{B}{D}\right)^{0.52} \times e^{-0.65 \times \frac{H}{B}} \\
& R_{t b}=\sigma_{t} \times B \times L \times\left(\frac{B}{D}\right)^{0.40} \times e^{0.84 \times \frac{H}{B}}
\end{aligned}
$$

where $\sigma_{c}, \sigma_{t}$ are the macroscopic compressive and tensile strength of the bonding material; $L$ is the length of the bond in the direction perpendicular to the paper; $D$ is the common diameter of the two disks with diameters $D_{1}$ and $D_{2}: D=2 D_{1} D_{2} /\left(D_{1}+D_{2}\right)$.

The projection of the envelope in $F_{s}^{b}-M^{b}, F_{s}^{b}-F_{n}^{b}$ and $M^{b}-F_{n}^{b}$ planes can be expressed as: 


$$
\begin{gathered}
\frac{F_{s}^{b 2}}{R_{s b}^{2}}+\frac{M^{b 2}}{R_{r b}^{2}}=1 \\
R_{s b}=0.34 \times \frac{B}{D} \times R_{c b} \times f \times\left[1+2 \times \ln \left(\frac{1}{f}\right)\right] \\
R_{r b}=0.1 \times B \times R_{c b} \times f \times\left[1+2 \times\left(\ln \frac{1}{f}\right)\right]
\end{gathered}
$$

where $f=\left(F_{n}^{b}+R_{c b}\right) /\left(R_{c b}+R_{t b}\right)$ is defined as the stress ratio, which can be used to define the type of bond failure: when $f=0$, compressive failure occurs; when $0<f<1$, tensile-shear-torsional (TST) failure $\left(F_{n}<0\right)$ or compressive-shear-torsional (CST) failure $\left(F_{n}>0\right)$ occurs; when $f=$ 1 , tensile failure occurs. If $u_{n}-u_{n 0} \geq 0$, the post-failure mechanical behavior can be expressed as:

$$
\begin{gathered}
F_{n}^{b}=\lambda R_{c b} \\
F_{s}^{b}=\min \left[K_{s}^{b} u_{s}, 0.34 \frac{B}{D} R_{c} f\right] \\
M^{b}=\min \left[K_{m}^{b} \theta, 0.1 B R_{c} f\right]
\end{gathered}
$$

where $\lambda$ is evaluated between 0 and 1 . Otherwise, the contact forces drop to zero.

\section{Numerical simulation of a direct shear test on jointed rocks}

\subsection{Intact rock material}

Calcarenite, a typical kind of soft rock (Ciantia et al., 2013) is chosen as the target material for the DEM simulation. In order to obtain such an intact rock material in DEM modeling, the procedure of parametric calibration, characterized by two classic experiments -Brazilian and uniaxial compression tests-, has been finished before the simulation of the direct shear test of jointed rock samples. Before these two tests, a homogenous sample is generated by the Multilayer with Under-compaction Method (Jiang et al., 2003), with an initial void ratio of 0.20 and the particle diameter distribution shown in Fig. 2. The final values of these microscopic bond contact model parameters are listed in Table 1. There are overall 8 parameters: four of them regarding the particle: $k_{n}$ and $k_{s}$ are the normal and shear stiffness; $\mu_{p}$ and $\beta_{p}$ are the friction and rolling resistance coefficient of particles. The other four are in relation to the cementing material: $E_{b}$ is the Young's modulus of bonding material; $s p_{b}=B / D$ is the ratio of the bond layer width to the diameter of the particle; $\sigma_{c}$ and $\sigma_{t}$ are the macroscopic compressive and tensile strength of the cementing material. The macroscopic mechanical properties of this DEM rock material, compared with the target soft rock material are presented in Table 2. 


\subsection{Simulation of direct shear test on echelon rock joints}

Prior to the DEM simulation of direct shear test on echelon rock joints, a $21.9 \mathrm{~cm} \times 13.1$ cm DEM rock sample composed of 30000 particles was generated in the same way as it was mentioned in the previous sub-section (Fig. 3). Joints are identified as layers of particles -within the rock sample- with a thickness of four times the minimum particle diameter. Joints are assigned with microscopic contact properties (shown in Table 1) including no bond effect. The two-dimensional rock joint constellations are characterized by three geometrical parameters, i.e. the inclination angle $i$ (the angle between the joint and negative direction of the horizontal axis, positive if the angle is clockwise), the length $l$ and the spacing $e$. Since the key point of this paper is to analyze the time-dependency of breakage within the jointed rock during the shear process, the influence of these geometrical parameters and loading conditions will be further discussed in another future academic paper. In this simulation, the inclination angle, the scale and degree of separation $\mathrm{l} / \mathrm{e}$ and the normal stress are $45^{\circ}, 0.6$ and $0.1 \mathrm{MPa}$, respectively. As for the time-dependency, four weathering scenarios, characterized by the ratio of the dissolved bond mass to the initial mass $\alpha$ (i.e. the time-dependent parameter), are described in the following section. During the direct shear tests, a constant normal stress was maintained by the horizontal rigid walls \#1 and \#4, and a shear load was applied to the bonded granular assembly by moving the vertical rigid walls \#2 and \#3 relatively to each other at a constant quasi-static velocity.

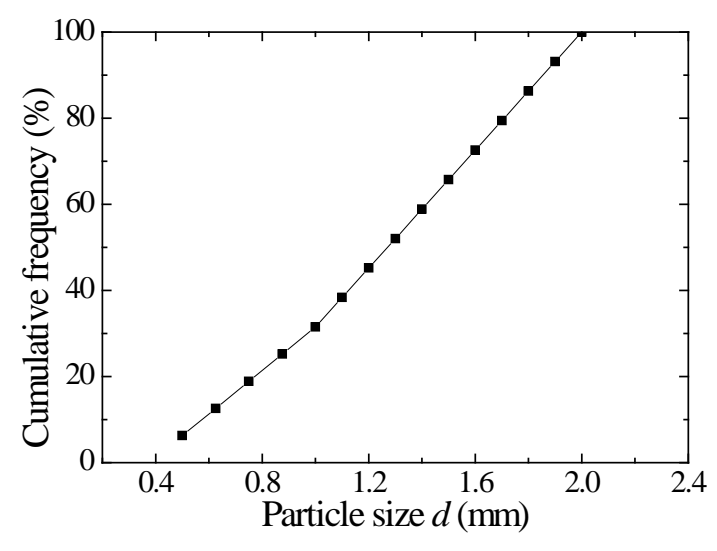

Figure 2. DEM samples: particle size distribution

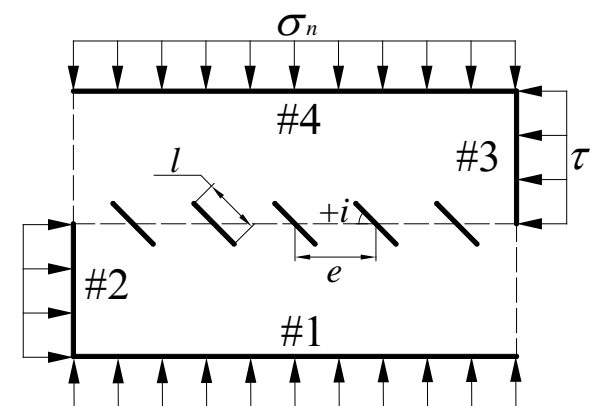

Figure 3. Shear specimen with intermittent joints 
Table 1. Parameters of the contact model of DEM analyses

\begin{tabular}{cc}
\hline Parameter & Value \\
\hline \multicolumn{2}{c}{ Particle } \\
\hline Density $\rho\left(\mathrm{kg} / \mathrm{m}^{3}\right)$ & 2700 \\
Normal stiffness $k_{n}(\mathrm{~N} / \mathrm{m})$ & $3.0 \times 10^{8}$ \\
Shear stiffness $k_{s}(\mathrm{~N} / \mathrm{m})$ & $1.2 \times 10^{8}$ \\
Friction coefficient $\mu_{p}$ & 1.0 \\
Anti-rotation coefficient $\beta_{p}$ & 1.5 \\
\hline \multicolumn{2}{c}{ Cementing material } \\
\hline Modulus $E_{b}\left(\mathrm{~N} / \mathrm{m}^{2}\right)$ & $8.27 \times 10^{6}$ \\
Compressive strength $\sigma_{c}$ & $232.4 \times 10^{6}$ \\
$\left(\mathrm{~N} / \mathrm{m}^{2}\right)$ & $16.6 \times 10^{6}$ \\
Tensile strength $\sigma_{t}\left(\mathrm{~N} / \mathrm{m}^{2}\right)$ & 0.15 \\
Spreading ratio $s p_{b}$ &
\end{tabular}

Table 2. The macroscopic properties of the intact soft rock material.

\begin{tabular}{ccc}
\hline Properties & Target & DEM \\
\hline Young's modulus $E(\mathrm{GPa})$ & 0.28 & 0.28 \\
Poisson ratio $v$ & 0.3 & 0.29 \\
Compressive strength $\sigma_{\mathrm{c}}(\mathrm{MPa})$ & 2.16 & 2.23 \\
Tensile strength $\sigma_{\mathrm{t}}(\mathrm{MPa})$ & 0.33 & 0.32 \\
\hline
\end{tabular}

\section{Results}

In this section, the time-dependency of the failure of the echelon rock joints during a direct shear test is analyzed in three aspects: 1) macroscopic shear behavior presented with microscopic bond failure numbers, 2) crack initiation shear and peak stresses and 3) post-failure configurations of the rock joint constellations. In this study, the time-dependent parameter $\alpha$ has been given four different values: $0 \%, 5 \%, 10 \%$ and $20 \%$, considering the potential slight chemical weathering condition in the practical rock engineering projects.

Figure 4 shows the relationships between shear stress (and microscopic bond failure numbers) and shear displacement during the shear test on the DEM jointed rock samples under varied weathering circumstances. All these four samples share the same shear behavior composed of four typical stages, i.e. elastic shearing phase, crack propagation, failure of rock bridge and through-going discontinuity. During the elastic shearing phase, no microscopic bond failure happens. When the microscopic bond failures occur, the cracks initiate then propagate, and eventually transect the material bridges with the peak shear stress reached. Finally, a through-going discontinuity appears and cuts through the set of the inclined echelon joints, represented by the drop of the shear stress from the peak to a lower residual strength. During the shear process, TST failures dominate the microscopic bond failures, which experience a sharp increase as the shear stress reaches the peak and then stay rather stable during the postpeak residual stage. 
(a)

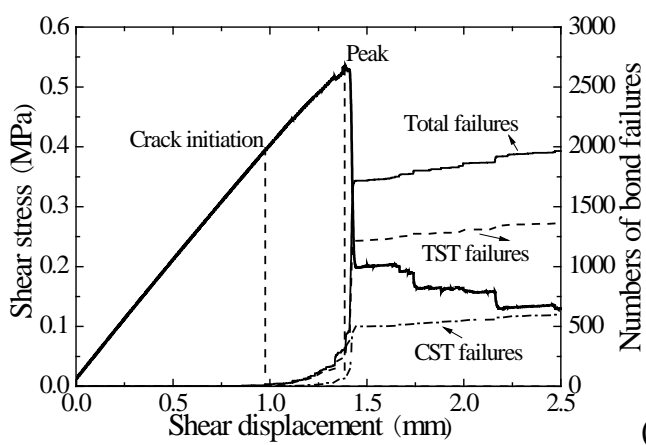

(b)

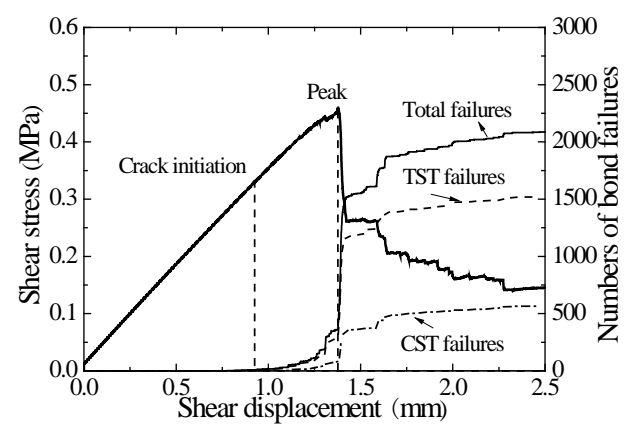

(c)

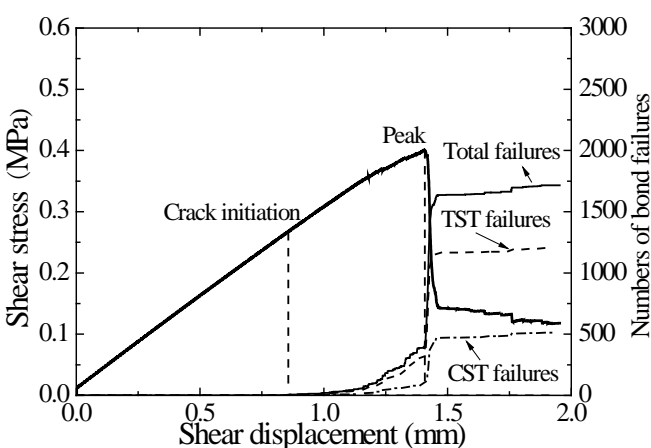

(d)

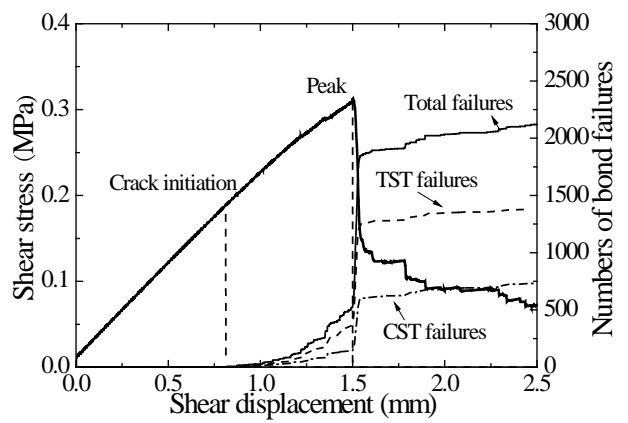

Figure 4. Relationships between shear stress (and microscopic bond failure numbers) and shear displacement with different $\alpha$ : (a) $0 \%$, (b) $5 \%$, (c) $10 \%$ and (d) $20 \%$

Figure 5 illustrates the shear loading capacity of the echelon joints with different $\alpha$. Both the crack initiation stress and the peak stress decrease with the increasing $\alpha$, which can prove that the microscopic alterations of the bonding material can result in great changes of the macroscopic mechanical responses.

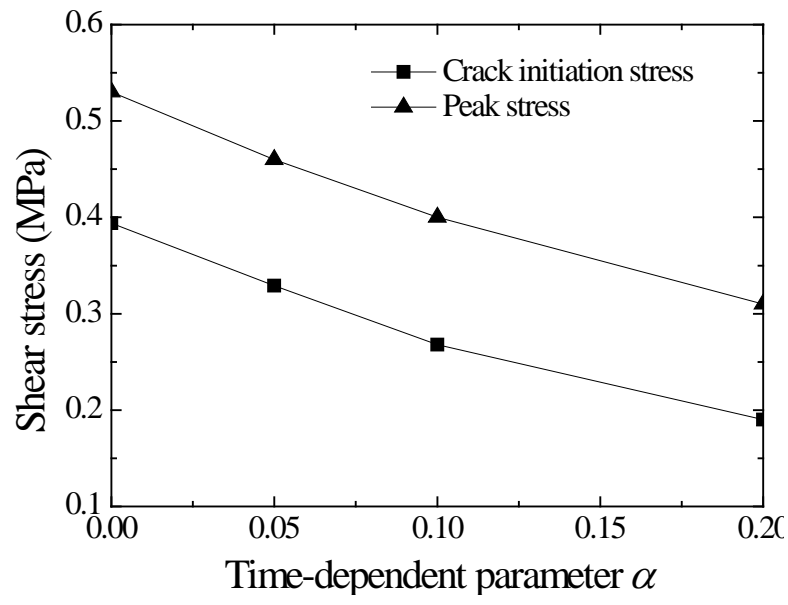

Figure 5. Shear loading capacity of the echelon joints with different $\alpha$

Figures 6, 7, 8 and 9 present the post-failure configurations of echelon rock joints in terms of force chains, principal stress vectors, bond and bond breakages. From Figures 6 and 7, it can 
be seen that the major principal stress is almost perpendicular to the orientation of the joints, and the parameter $\alpha$ has a great impact on the stress distribution among those material bridges, e.g. a larger $\alpha$ makes the stress concentrate into a smaller part of the bridge, even though the bond degradation is homogenously distributed within the rock sample. Figures 8 and 9 show the bond breakage among these rock joints. With a higher accumulated relative mass removal of the bond, $\alpha$, the material bridge between parallel joints tends to be transect from the mid of the bridge, forming a complete discontinuity along the central shear axis. While for those with lower $\alpha$, some wing cracks initiate from the tip of the joint and develop towards the adjacent tip of the neighboring joint. In Figure 9, the bond breakages of both CST (blue) and TST (red) failure types are shown within the jointed rock sample, reaffirming the bond failure numbers presented in Figure 4. Most of the CST bond breakages are located along the central shear axis, especially for the case with higher $\alpha$.

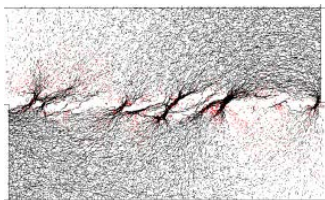

(a) $\alpha=0 \%$

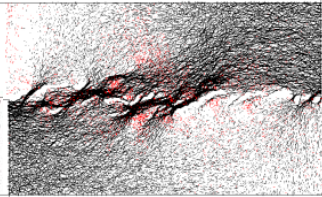

(b) $\alpha=5 \%$

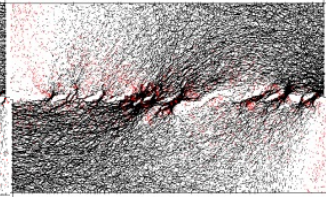

(c) $\alpha=10 \%$

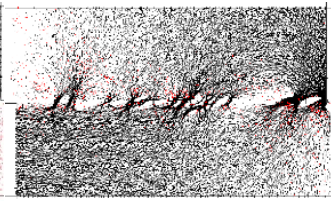

(d) $\alpha=20 \%$

Figure 6. Force-chain distributions of four cases with different $\alpha$

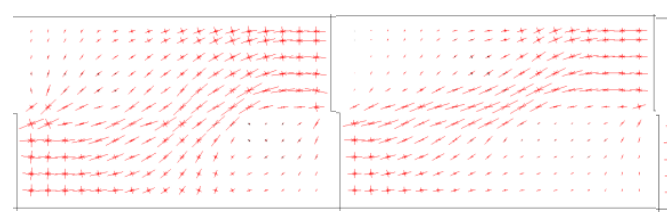

(a) $\alpha=0 \%$

(b) $\alpha=5 \%$

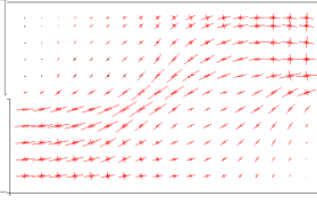

(c) $\alpha=10 \%$

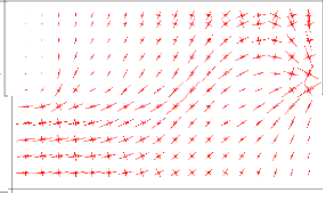

(d) $\alpha=20 \%$

Figure 7. Principal stress vector fields of four cases with different $\alpha$

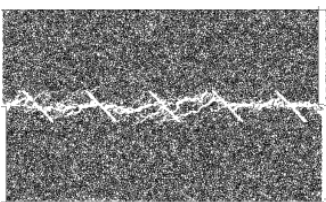

(a) $\alpha=0 \%$

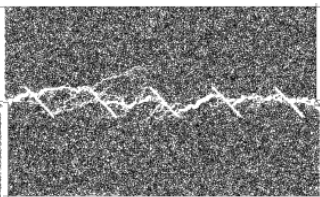

(b) $\alpha=5 \%$

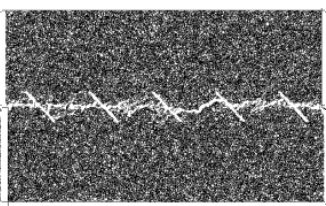

(c) $\alpha=10 \%$

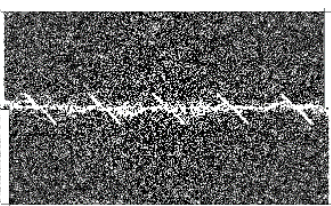

(d) $\alpha=20 \%$

Figure 8. Bond distributions of four cases with different $\alpha$

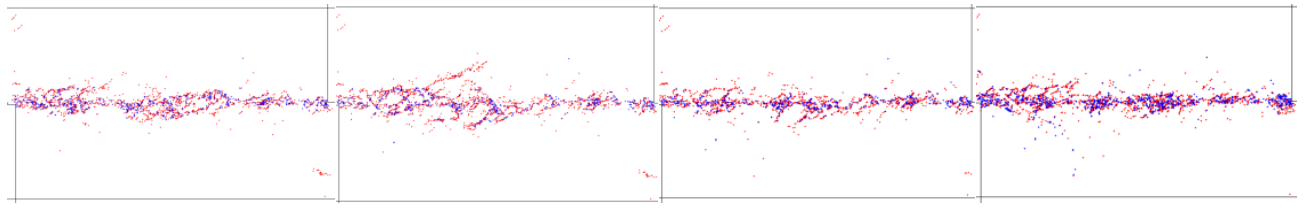
(a) $\alpha=0 \%$
(b) $\alpha=5 \%$
(c) $\alpha=10 \%$
(d) $\alpha=20 \%$

Figure 9. Bond breakage distributions of four cases with different $\alpha$ 


\section{Conclusions}

This paper analyzes the time-dependent behavior of parallel rock joints in the echelon arrangement caused by slight chemical weathering through introducing a time-dependent parameter identifying the accumulated relative mass removal of the bond material cementing rock grains. This debonding law has been implemented into the DEM bond contact model on the basis of the experimental data extracted from mechanical test on bonded aluminum rods with different bond geometries. The main conclusions are as follows: the shear behaviors of samples with different time-dependent parameters are similar, except for some characteristic values such as crack initiation and peak shear stress. Both of them decrease with the growing time-dependent parameter $\alpha$. A higher $\alpha$ always makes the material bridge weaker to assume the loading transferred though it, rendering it easier to be transected by the crack initiating from the mid of the parallel joints. From the microscopic level, the ratio of CST bond breakage number to the total one increases with the growing $\alpha$ except for the non-weathered case, which means that the material bridge with higher $\alpha$ is biaxial compressive state-like instead of Brazilian test-like loading state for cases with lower $\alpha$. Overall, the DEM simulation characterized by a novel bond model has been proved to be a feasible tool to numerically investigate the time-dependent behavior of the rock material.

\section{References}

Buscarnera, G. (2012). A conceptual model for the chemo-mechanical degradation of granular geomaterials. Geotechnique Letters, 2, 149-154.

Chigira, M., \& Oyama, T. (2000). Mechanism and effect of chemical weathering of sedimentary rocks. Developments in geotechnical engineering, 84, 267-278.

Ciantia, M., Castellanza, R., \& di Prisco, C. (2013). Chemo-mechanical weathering of calcarenites: experiments and theory. Coupled phenomena in environmental geotechnics. Taylor \& Francis Group, London, 541-548.

Ciantia, M., \& Hueckel, T. (2013). Weathering of submerged stressed calcarenites: chemo-mechanical coupling mechanisms. Géotechnique, 63(9), 768-785.

Dusseault, M. B., \& Fordham, C. J. (1993). Time-dependent behavior of rocks. Comprehensive Rock Engineering Principles, Practice and Project: Rock Testing and Site Characterization, 3, 119-149.

Duzgoren-Aydin, N., Aydin, A., \& Malpas, J. (2002). Re-assessment of chemical weathering indices: case study on pyroclastic rocks of Hong Kong. Engineering Geology, 63(1), 99-119.

Gehle, C., \& Kutter, H. (2003). Breakage and shear behaviour of intermittent rock joints. International Journal of Rock Mechanics and Mining Sciences, 40(5), 687-700.

Ghazvinian, A., Sarfarazi, V., Schubert, W., \& Blumel, M. (2012). A study of the failure mechanism of planar non-persistent open joints using PFC2D. Rock Mechanics and Rock Engineering, 45(5), 677-693. Hachinohe, S., Hiraki, N., \& Suzuki, T. (2000). Rates of weathering and temporal changes in strength of bedrock of marine terraces in Boso Peninsula, Japan. Engineering Geology, 55(1), 29-43.

Jiang, M., Konrad, J., \& Leroueil, S. (2003). An efficient technique for generating homogeneous specimens for DEM studies. Computers and Geotechnics, 30(7), 579-597. 
Jiang, M., Yu, H., \& Harris, D. (2006). Bond rolling resistance and its effect on yielding of bonded granulates by DEM analyses. International Journal for Numerical and Analytical Methods in Geomechanics, 30(8), 723-761.

Ohta, T., \& Arai, H. (2007). Statistical empirical index of chemical weathering in igneous rocks: A new tool for evaluating the degree of weathering. Chemical Geology, 240(3), 280-297.

Price, J. R., \& Velbel, M. A. (2003). Chemical weathering indices applied to weathering profiles developed on heterogeneous felsic metamorphic parent rocks. Chemical Geology, 202(3), 397-416.

Sarfarazi, V., Ghazvinian, A., Schubert, W., Blumel, M., \& Nejati, H. R. (2014). Numerical Simulation of the Process of Fracture of Echelon Rock Joints. Rock Mechanics and Rock Engineering, 47(4), 13551371.

Tang, C., Yang, W., Fu, Y., \& Xu, X. (1998). A new approach to numerical method of modelling geological processes and rock engineering problems-continuum to discontinuum and linearity to nonlinearity. Engineering Geology, 49(3), 207-214.

Velbel, M. A. (1993). Constancy of silicate-mineral weathering-rate ratios between natural and experimental weathering: implications for hydrologic control of differences in absolute rates. Chemical Geology, 105(1), 89-99.

White, A. F., \& Brantley, S. L. (2003). The effect of time on the weathering of silicate minerals: why do weathering rates differ in the laboratory and field? Chemical Geology, 202(3), 479-506.

Wong, R., Leung, W., \& Wang, S. (2001). Shear strength studies on rock-like models containing arrayed open joints. Paper presented at the DC Rocks 2001 The 38th US Symposium on Rock Mechanics (USRMS).

Wong, R. H., \& Chau, K. (1998). Crack coalescence in a rock-like material containing two cracks. International Journal of Rock Mechanics and Mining Sciences, 35(2), 147-164.

Zhang, H., Zhao, Z., Tang, C., \& Song, L. (2006). Numerical study of shear behavior of intermittent rock joints with different geometrical parameters. International Journal of Rock Mechanics and Mining Sciences, 43(5), 802-816. 